\title{
Influencias cognoscitivas de la tecnología de información y comunicación en el aprendizaje de la matemática
}

\section{Cognitive influences of information and communication technology in the learning of mathematics}

\author{
Derling Jose Mendoza Velazco \\ Universidad Iberoamericana del Ecuador-UNIB.E, Ecuador
}

Autor corresponsal: dmendoza@ unibe.edu.ec

Fecha de recepción: 04 de Enero de 2018 - Fecha de aceptación: 15 de Mayo de 2018

\section{Resumen}

En el presente artículo se organiza un análisis introspectivo vivencial del aprendizaje significativo, mediante los resultados de una investigación cualitativa, estructurada bajo los paradigmas de la fenomenología interpretativa y la innovación tecnológica, como pilares de enlace primordial para proceder en la indagación del objeto de estudio, estas deducciones de razonamiento aportan nuevas maneras de forjar y vivir la educación, por medio de la aplicación coherente de las tecnologías de información y comunicación (TIC), facilitando y permitiendo la concepción del desarrollo cognitivo, para el pensamiento lógico matemático de los estudiantes en la Universidad Iberoamericana del Ecuador UNIB.E.

Palabras Claves: aprendizaje cognoscitivo; TIC; educación; matemática

\begin{abstract}
In this article we organize an introspective experiential analysis of meaningful learning, through the results of a qualitative research, structured under the paradigms of interpretive phenomenology and technological innovation, as pillars of primordial link to proceed in the investigation of the object of study, These deductions of reasoning provide new ways of forging and living education through the coherent application of information and communication technologies (ICT), facilitating and allowing the conception of cognitive development, for the logical thinking of students at the level academic.
\end{abstract}

Key words: cognitive learning; ICT; education; mathematics 


\section{Introducción}

Para el hombre la investigación es una actividad principal desde el inicio de los tiempos. El anhelo de aprender, conocer, observar y la necesidad de analizar para movilizarse en un mundo tanto predecible como interpretativo, es desde hace muchos años una variable del quehacer humano. La educación tradicional en gran medida privilegia las capacidades repetitivas e imaginativas más que las creativas del docente y el estudiantado, incorporar las TIC al proceso de investigación, representa cambios significativos generalizando un paso evolutivo importante y una innovación que facilita con mayor fluidez de encuentros cercanos entre especialistas y estudiantes geográficamente, pero conectados a la amplia red socio educativa y virtual del ciberespacio, que proporciona oportunidades de desarrollo investigativo.

Los métodos tradicionales de enseñanza y aprendizaje en las matemáticas, no presentan efectividad a los educandos, ya que estas formalizan anomalías consecuentes que son críticas comunes por los adolescentes, como el temor o rechazo que tienen de aversión a la matemática. La investigación en ciencias cognitivas proporciona evidencias empíricas que muestran que el conocer no es un medio pasivo de una información, por el contrario, la implementación de la tecnología para el ámbito universitario, juega un papel importante en el funcionamiento intelectual, para el procesamiento de información que recibe, dichos procesos son en gran medida, los responsables de conocimientos que se adquiere.

Del mismo modo, se puede señalar que las corrientes psicológicas cognoscitivas, fomentadas con la aplicación de TIC, alcanzan el predominio en el campo investigativo del aprendizaje humano, acumulando evidencias suficientes, para afirmar que la mayoría de los estudiantes de matemática a nivel superior, necesitan laborar con modelos y representaciones digitales, para un aprendizaje significativo del deber ser, y así adquirir de manera sencilla un conocimiento abstracto, complejo y simbólico de la ciencias numéricas. Los resultados de la investigación podrían ser útiles en otras áreas del conocimiento porque contribuye con análisis y orientaciones en distintos niveles del desempeño educativo profesional.

\section{El conocimiento}

Para el ser humano el conocimiento es una actividad esencial para adquirir información, desarrollar la vida y certezas de la realidad. Todo discernimiento requiere forzosamente una relación en la cual aparecen dos elementos vinculados entre sí: el individuo y el objeto. Landeau (2012), define que:

El conocimiento es un conjunto de información que posee el ser humano del escenario que lo rodea y de sí mismo, valiéndose de los sentidos y de la reflexión para obtenerlo; luego lo utiliza como material para precisar las características de los objetos en su entorno empleando la observación. (p. 1)

El hombre como ser complejo y de variados razonamientos, por lo tanto, tiene diferentes formas de acercarse y adquirir información y resolver problemas. Varios autores centralizan el conocimiento en dos aristas únicas y clasificatorias, entre el conocimiento científico y el vulgar, pero también se debe tipificar según las distintas inclinaciones al percibir la realidad, entre ellos el común, el cual es adquirido mediante la experiencia y contacto con la vida. El religioso, que 
procede de la revelación profética de las tradiciones o libros sagrados, de Dios o de dioses. El conocimiento social, que trata de una acción instrumental, de una depuración de toda alteridad, que conduce a la producción semántica organizadas a partir de constructores basados en la lógica.

El conocimiento filosófico, proviene de la introspección sistémica y metódica de las últimas realidades de la existencia humana, se encuentra sólidamente abierto al reconocimiento y es habitual para la exploración y visión de los fenómenos de las investigaciones. El científico en general es considerado como el más difundido, ya que proporciona teorías con un sistema que desarrolla acuerdos con la naturaleza y comunidades de pensamiento metodológico. Es una de las maneras reflexivas que tiene el individuo de otorgarle un significado a la vida. El más adecuado para dar respuestas y soluciones a gran cantidad de interrogantes humanas a través de la explicación, aplicación y demostración metódica y técnica.

\section{La psicología cognoscitiva}

La filosofía se ocupó durante mucho tiempo del estudio del conocimiento implicando teorías acerca del mismo, entre estas la del racionalismo, sus exponentes fundamentan la posibilidad del conocimiento en la realidad inteligible, es decir entre la idea y la razón. El idealismo moderno coincide con el racionalismo, desarrollando el idealismo filosófico de Kant. Wundt (1879), en su publicación "principios de la psicología fisiológica" expone que la mente humana debe ser estudiada detalladamente en forma experimental y objetiva. Su enfoque investigativo se resume en la psicología como ciencia de la experiencia inmediata y su método es el introspeccionismo.

La escuela de Wundt se le conoce con el nombre de instrospeccionismo, su objetivo es descubrir las leyes de la mente humana, mediante la introspección, para ello se entrenaba a los estudiantes en el uso de la observación objetiva, de sus reacciones, percepciones, sensaciones y llevar record cuidadoso de ellos. Al contrario, para Freud (1915), sostiene que la experiencia psicológica no solo es una experiencia consciente, sino también inconsciente. Luego se presentaron las contradicciones del conductismo, que proclama que el objeto de psicológica cognitiva no es la introspección, sino la conducta y posteriormente de la psicología de Gestalt (1930), para quien la conducta no se puede estudiar fragmentada, es decir, sumando percepciones y sensaciones, sino como una totalidad.

Para Gestatlt (1930), El fenómeno del aprendizaje está estrechamente ligado a la percepción, en consecuencia, define el aprendizaje de acuerdo con la reorganización del mundo perceptual individual. Herbert (1979), define la percepción como "un fenómeno cognoscitivo, por el cual, a través de la estimulación de los órganos de los sentidos, se experimenta la presencia de los objetos del mundo exterior" (p. 12). Estas características que se presentan en una situación de aprendizaje, son de extraordinaria importancia, debido a que el organismo es totalmente activo y su acción es la de organizar, construir lo que se recibe del medio ambiente (estímulos), considerándose la percepción como un proceso unilateral, donde la percepción y la determinación del significado se producen simultáneamente.

\section{Estructura cognoscitiva}


El término estructura cognoscitiva, es considerado importante como factor que influye en el aprendizaje y garantiza, la retención significativa de los nuevos conocimientos. Según Pírela, Gómez y González (2002), "la estructura cognoscitiva se refiere al contenido total y a la organización de las ideas que un individuo posee en cualquier área del conocimiento" (p. 468). De la definición se deduce, que la estructura cognoscitiva debe poseer como característica fundamental, la claridad y organización, para que pueda surgir significados precisos pertinentes a la nueva información recibida y garantizarse así la comprensión e internalización de los nuevos conocimientos.

En el aprendizaje significativo de Ausubel, la estructura cognoscitiva constituye siempre una variable decisiva, que tiene que ver con la transferencia, es decir, la interrelación que se produce entre los nuevos conocimientos y la estructura cognoscitiva existente. Las transferencias se refieren al efecto de la experiencia previa, sobre el aprendizaje presente; pero en este caso para el estudiante su conocimiento anterior se conceptúa como una fuente de datos preestablecida, organizada jerárquicamente y adquirida en forma acumulativa.

\section{Desarrollo cognoscitivo}

Ausubel considera importante los cambios, distinguiéndolos como diferentes etapas evolutivas en el desarrollo cognoscitivo, por cuales pasa el niño hasta la adolescencia. La etapa pre-operacional, de distingue por la adquisición de conceptos primarios, los cuales construyen a partir de la experiencia directa con el medio ambiente y con los objetos. La etapa de operaciones concretas; el niño es capaz de adquirir conceptos secundarios y de comprender, emplear y manejar significativamente abstracciones secundarias como relaciones entre estas. La etapa lógica-abstracta, se caracteriza por un pensamiento distante y alejado de los hechos relativos al mundo real, basado más a un razonamiento hipotético deductivo.

Al respecto Ausubel (1976), plantea "En lugar de razonar basado directamente en un conjunto particular de datos, recurre a operaciones lógicas indirectas, de segundo orden, para estructurar los datos...; formula y prueba hipótesis basadas en todas las combinaciones posibles de variables" (p. 240). Esta etapa se ubica en la adolescencia y al inicio de la educación media general, lo que verdaderamente distingue según Ausubel, el pensamiento del niño y del adolescente en este nivel, es la capacidad para manejar relaciones en forma verbal, sin apoyo en las relaciones concretas. Los conceptos y generalizaciones que se establecen se derivan de relaciones entre complejidades y abstracciones verbales previamente establecidas. Es decir, puede adquirir conceptos a través de definiciones.

\section{Aprendizaje conectivista}

Para Siemens (2004), citado por Calle (2011), teoriza que:

El punto de partida del conectivismo es el individuo. El conocimiento personal se compone de una red, la cual alimenta a organizaciones e instituciones, las que a su vez retroalimentan a la red, proveyendo nuevo aprendizaje para los individuos. Este ciclo de desarrollo del conocimiento (personal a la red, de la red a la 
institución) les permite a los aprendices estar actualizados en su área mediante las conexiones que han formado. (p. 190).

La teoría del conectivismo se presenta como un tipo de aprendizaje que registra los movimientos pragmáticos de la sociedad, en la cual el individuo no obtiene el conocimiento de forma individual o personalizada desde su interior. Por lo tanto, la manera como laboran y actúan los compañeros aturde en general, al emplearse nuevas herramientas. En el ámbito educativo, los docentes presentan la resistencia a los cambios ambientales que favorecen la aplicación de las TIC. Como teoría actual de la era digital, prevé de grandes oportunidades que facilitan al estudiante para desarrollar habilidades de aprendizaje.

\section{Aprendizaje significativo} los datos:

Ausubel, Novak, y Hanesian (1983), afirman que el aprendizaje es significativo cuando

Son relacionados de modo no arbitrario y sustancial (no al pie de la letra) con lo que el alumno ya sabe. Por relación sustancial y no arbitraria se debe entender que las ideas se relacionan con algún aspecto existente específicamente relevante de la estructura cognoscitiva del alumno, como una imagen, un símbolo ya significativo, un concepto o una proposición. (p. 18).

El aprendizaje significativo es aquel en el cual el estudiante es capaz de relacionar los contenidos que se presentan en forma sustancial y no arbitraria a su estructura cognoscitiva. En forma sustancial existe la vinculación de lo esencial del conocimiento nuevo, a lo que el estudiante ya sabe. Como recurso básico, es necesario que los jóvenes posean conocimientos previos que hagan que este material potencialmente significativo, se puede agregar que igualmente interactúan dos factores, uno se relaciona con la estructuración cognoscitiva del estudiante y como segundo factor el material, empleado en la investigación como el recurso tecnológico.

\section{Las TIC en la educación}

En la era de la globalización, el uso de la tecnología es una de las aplicaciones más importantes en el campo de la educación, lo que ha permitido ganar un espacio legítimo en todo el contexto educativo en el ámbito mundial. En este orden de ideas, el uso de la tecnología en la Educación ha sido clave para el desarrollo y la creación de tecnologías educativas en la acción cotidiana en las aulas. Landeau (2012), define las TIC, como "herramientas computacionales e informáticas, que procesan, sintetizan, reivindican y presentan información representada de la forma más renovada. Este recurso es incuestionable, ya que forma parte de nuestra cultura tecnológica, nos rodea y con la cual debemos convivir”. (p. 130). La implementación de nuevas tecnologías se ha desarrollado en paralelo con los cambios en los métodos de enseñanza y aprendizaje, ya que permiten incrementar capacidades físicas y mentales a los estudiantes, como también las posibilidades de desarrollo socio educativo.

Para Fainholc (2012), establece que: 
La tecnología educativa debe responder a las necesidades específicas de las sociedades en las cuales habrá de funcionar, debe ser pertinente, debe tener presencia en las políticas públicas, adaptarse a los sistemas sociales y culturales, a los intereses lingüísticos de los grupos receptores participantes. (p. 16).

Por consiguiente, los docentes han encontrado un mundo de posibilidades para el desarrollo de su práctica pedagógica a través de integrar las TIC como un recurso más en el proceso de enseñanza - aprendizaje, que les ha permitido promover y facilitar la actitud participativa y creadora de los estudiantes(as), la enseñanza individualizada del aprendizaje interactivo, la formación a distancia y de nuevas metodologías generadoras de conocimientos como objeto de investigación, en el desarrollo cognitivo apoyado por computadora, lo que ocasiona una verdadera transformación en el proceso de enseñanza y aprendizaje al ceder el papel protagónico al estudiante.

\section{La educación matemática}

El momento histórico que está siendo protagonizado por la humanidad entera, está dominado por el uso de las TIC, las cuales sirven de base a la mundialización de la economía, e impactan toda la cotidianidad vital de las personas a lo largo y ancho de nuestro país; esto implica demandas cognitivas inusitadas y al mismo tiempo, procesos de desaprendizaje, reaprendizaje y aprendizaje de novedosos conceptos que hagan viable la instalación de competencias en todas las personas de modo que puedan desenvolverse adecuadamente en los nuevos contextos sociales que caracterizan a las actuales relaciones sociales, económicas, políticas y culturales en un ámbito globalmente generalizado.

En este marco de referencia, a la Matemática se le atribuye un papel protagónico; por ende, el dominio de esta disciplina no debe seguir siendo conocimiento exclusivo de unas pocas personas, sino que, por el contrario, pasa a ser una exigencia cultural indispensable para todo ciudadano. En efecto, como puede verificarse al examinar la historia de la educación, desde la academia de Platón y el Cuadrivium Romano, a los estudiantes se les ha requerido el estudio de la matemática para que aprendan a razonar claramente. También es posible apreciar que los adelantos técnicos y de otro tipo que han marcado la historia de la humanidad han estado vinculados, en alguna forma, con el desarrollo de esta ciencia.

Por lo tanto, se ratifica la necesidad social del estudio de esta disciplina y cómo, a través del tiempo, se ha reconocido su relevancia. Con base en lo anterior, podría decirse que el conocimiento de esta asignatura, alcanza el rango de derecho humano inalienable. Ahora bien, tomando en cuenta que los estudiantes que hoy están en los niveles de educación secundaria son los futuros dirigentes de una sociedad que, cada vez más, estará caracterizada por una economía global y sumergida en el marco de un uso creciente de las TIC, cuyo sustrato de desarrollo es la Matemática, en este momento resulta, vitalmente estratégico que aprendan esta disciplina en función de las exigencias que les hará el entorno científico y tecnológico del mañana.

\section{Desarrollo cognitivo y socio emocional de las TIC}


Las TIC transportan mensajes según lenguajes o códigos simbólicos específicos. Es necesario definir a los estudiantes como perceptores activos, y no como receptores pasivos de dichos mensajes. Es decir, son aquellos que han desarrollado competencias audiovisuales y mediáticas, además de digitales, que les permiten percibir de modo individual y grupal, en forma y contenido, los mensajes presentados en formatos y soportes de los medios convencionales. Para ello se reconoce que una competencia comunicativa, engloba y articula el desempeño de las competencias cognitivas, estableciendo un conjunto complejo de saberes conceptos, procedimientos y actitudes puestas en práctica reflexiva y contrastada en una situación de comunicación mediada por tecnología.

Los actores deberán desplegar críticamente su expresión de investigación dentro de una situación comunicacional particular. Según Cabero (2005):

... la fragmentación de las disciplinas, que hará que los límites entre las disciplinas sean más difuso que los actuales y nos llevará a la transformación de las áreas de conocimiento; se pasará de modelos centrados en el profesor, a modelos centrados en el estudiante, y de modelos donde lo importante sea la enseñanza a modelos que giren en torno al aprendizaje de habilidades, contenidos y competencias por los estudiantes; y el hecho de que éstos deberán adquirir nuevas competencias y capacidades, destinadas nos sólo al dominio cognitivo, sino también en sus capacidades para aprender, desaprender y reaprender, para adaptarse a las nuevas exigencias de la sociedad. (p. 6)

Estas adaptaciones significan, emplear una combinación de recursos humanos, materiales y acciones para conseguir un aprendizaje más efectivo. De igual manera, es aquella que reflexiona sobre la aplicación de la técnica a la aplicabilidad de actividades educativas, justificada en la ciencia vigente de cada momento histórico. A tal efecto, enfatiza el control del sistema de enseñanza y aprendizaje como aspecto central y garantía de calidad, a la vez que entiende que las opciones más importantes están relacionadas con el tipo de técnica que conviene y cómo incorporarla adecuadamente. Es decir, a la utilización de las TIC en el campo de la enseñanza, se enlaza a un mismo vocablo. Por consiguiente, la tecnología se considerada como un lenguaje que expresa la capacidad del ser humano para utilizar y combinar articuladamente procedimientos, medios que les permiten formalizar una competencia mediática.

En este sentido, la tecnología educativa es el lenguaje que tiene que ver directamente con ambas competencias, la comunicativa y la mediática, se inscriben previamente en el dominio de las competencias digitales. Los contextos audiovisuales, las redes y las TIC en general permiten prácticas y expresiones de diverso tipo, como investigar, transcribir, leer, redactar, analizar, criticar, etc. Este poderoso elemento digital facilita el acceso a caudales enormes de información distribuida, los cuales optimiza el interés de los estudiantes en la actualidad, potenciando el desarrollo del libre pensamiento para la producción y publicación, uso y discusión del conocimiento elaborado, hasta constituirse una meta de cualquier programa formativo, presencial y a distancia, formal, no formal e informal.

Gardner (2011), afirma que: 
Articular recursos tecnológicos para un uso inteligente, significa que demuestran ser satisfactorios porque son útiles, valiosos, viables, precisos, realistas, lúcidos, prudentes y éticos; y por ello se han incorporado al hardware, al software y al mindware o a tecnologías invisibles de la mente para una práctica social y educativa adecuada. En realidad, hoy más que nunca, deberían conformarse y convertirse en "programas de estimulación cognitiva" que tiendan a fortificar el desarrollo de una "cultura de pensamiento". (p. 57)

Siendo las inteligencias las capacidades humanas que posibilita establecer relaciones, constituye la problemática nuclear del desarrollo cognitivo y socioemocional de las personas. No sólo es menester detenerse en el interior del diseño, del desarrollo de las estrategias cognitivas, en consecuencia, en la meta cognición, sino que además es necesario explorar y aprender, de la práctica mediada por las TIC, el impacto producido en la mente. Ello es de real relevancia en la estructuración de la personalidad y por su proyección futura. El empleo de la fantasía para el estímulo de la imaginación, la creación de alternativas y la inclusión del humorismo, para suavizar o equilibrar las frustraciones de la vida contemporánea.

Para modificar y acrecentar el rendimiento cognitivo, metacognitivo y socioemocional, los medios y las mediaciones de las tecnologías habrán de desarrollar habilidades de pensamiento que optimicen las operaciones intelectuales y doten de instrumentos de análisis y esquemas de actividad para operar sobre la realidad, como consecuencia de una transferencia pertinente. Dichas habilidades deberán ser activadoras y facilitadoras de los procesos de percepción y de reelaboración crítica de la información, de un modo selectivo, lúcido y aplicativo. Es decir, los medios cumplirían así la función de soportes de las estructuras externas para estimular, conformar y reorganizar el pensamiento superior. Lo que se necesita fundamentalmente es estructurar oportunidades para pensar y para examinar los resultados de las aplicaciones. Por ello, habrá que crear situaciones para que los estudiantes resuelvan problemas y realicen experiencias que faciliten anticipar e inventar modificaciones para mejorar las prácticas sociales con el desarrollo de las diversas capacidades.

La interacción en la experiencia conjunta con otros de modo contrastado es lo que contribuye, del modo más significativo, al proceso de maduración y desarrollo cognitivo. A ello deben estar abocadas las producciones de las TIC y las interacciones de las redes, promoviendo situaciones que ejerciten el pensar y fortalezcan la comprensión. Por ello, se sostiene que, entre otras muchas funciones más, comparar, interpretar, observar y resumir son operaciones intelectuales, en el sentido de que su empleo inteligente, útil y valioso despierta y produce el pensamiento. Lo enunciado, unido a la toma de decisiones sobre tecnología educativa articulada con las TIC, a través de políticas públicas en múltiples arcas, conduciría al mejoramiento de la calidad educativa, en lo referido a comunicación y educación.

Asimismo, posee relevancia aprestar y ejercitar el pensamiento creador y la inventiva paso previo a anticipar innovaciones o pensar soluciones inéditas o alternativas a diversos problemas que es el desafío actual para los adolescentes del mundo virtual, acelerado e imprevisible, habrá que estimular la imaginación. Sin imaginación, la humanidad se hubiera estancado y jamás hubiera llegado a ser lo que es hoy, tanto en lo positivo como lo negativo, porque no se hubiera formado ideas de cosas no presentes a causa de no imaginar. El empleo de 
la fantasía en las producciones de los medios de comunicación, de las TIC e Internet constituye una vía efectiva y atractiva hacia la socialización futura, que afianza el desarrollo de los planos real e imaginario para su proyección y construcción, en este caso en las reales telemáticas.

Las investigaciones neurofisiológicas y psicológicas han demostrado la enorme plasticidad del cerebro, además de la importancia de su hemisferio derecho, que controla el pensamiento concreto, holista y artístico, donde reside la imaginación desde los primeros años de vida. Luego, desgraciadamente y debido a uniformidades varias, se va atrofiando por prevalencia unidireccional de las actividades lógicas, abstractas y formales, las que se imponen con las actividades sencillas desde la educación básica, entre otras interacciones. No se trata de hacer prevalecer uno sobre el otro, sino que el pensamiento abstracto e imaginación converjan en el proceso creativo, sobre todo, por el peso de las TIC con el uso de las imágenes, para desarrollar su complementariedad. Así, se formarán estudiantes con perspicacia, capaces de prever y afrontar cambios, inventar soluciones a problemas en las distintas esferas del arte, la ciencia, la cultura y la tecnología.

\section{La conexión entre la educación y lo cognitivo en las TIC}

Según Delors (1997), "El proceso emocional a lo interno de la comunidad es tan importante como el cognitivo". (p. 1). Para el autor las comunidades están representadas por los entes, organizados educativos, son procesos integrados y complementarios que forman un todo integrado llamado persona, que se compone, por las dimensiones del ser, el conocer, el hacer y el convivir. En las comunidades de aprendizaje existe una fuerte conexión entre lo social y lo cognitivo. Toma importancia las relaciones emocionales que se desarrollan entre los estudiantes.

Novak (1998) establece que:

Una educación acertada debe centrarse en algo más que el pensamiento del aprendiz; los sentimientos y las acciones también son importantes, y hay que tener en cuenta estas tres formas de aprendizaje, a saber: la adquisición de conocimientos (aprendizaje cognitivo), la modificación de las emociones y los sentimientos (aprendizaje afectivo) y la mejora de la actuación o las acciones físicas o motrices (aprendizaje psicomotor), que incrementa la capacidad de la persona para entender sus experiencias (...) Los seres humanos piensan, sienten y actúan, y las tres cosas se combinan para formar el significado de la experiencia. (p. 28)

Una comunidad de aprendizaje, por tanto, atiende al estudiante como ser integral ofreciendo experiencias retadoras en todas sus dimensiones. El eje conector de un grupo educativo es el placer de aprender con otros, y ello se valora en la presente investigación. La presencia social de cada integrante conforma un sentido global de los adolescentes, que a su vez favorece que los procesos cognitivos se desarrollen sin limitarse por las inseguridades o desconfianzas de algunos miembros. Los ambientes virtuales benefician las interacciones cognitivas, alineadas a la construcción de conocimiento en contribución, con predominio de niveles iniciales del pensamiento crítico. 
La aplicación de las TIC apunta hacia el desarrollo de interacciones en tres ámbitos: cognitivo, social y moderado. Si bien las interacciones sociales y tutoriales son fundamentales para el sentido de identidad, la participación y la orientación, se considera que ambas están al servicio del proceso cognitivo de los estudiantes en la institución, de las construcciones que puedan elaborar con base en la ayuda mediada a través del diálogo crítico, de la reflexión conjunta y del conocimiento de otras experiencias en su relación con otros compañeros y el docente. Para García (2010), "La interacción cognitiva está referida al desarrollo del pensamiento crítico". (p. 127). Se entiende como un proceso que inicia con una situación activadora, problemática, generadora de conflictos de índole cognitivo los cuales orientan al estudiante a la investigación e indagación de información, recuperación de experiencias vividas y suposición de ideas, permitiéndoles comprender la situación problemática, para la búsqueda de soluciones.

\section{El paradigma de la innovación tecnológica}

Para Cabada (2001), "Se reconocen una serie de características del paradigma tecnológico, entre ellas, el creciente papel de las innovaciones tecnológicas, el aumento de la demanda de información y nuevos conocimientos”. (p. 224). Las tipologías del universo investigativo describen la innovación tecnológica, dentro de un paradigma o patrón tecnológico, que explica anticipadamente la visión a partir de la cual se propone la solución a problemas, como vías de provocar un auténtico progreso tecnológico. Para la investigación la aplicación de proyectos tecnológicos pertinentes, la ejecución de competencias tecnológicas aprendidas y el uso óptimo e inteligente de artefactos con el objetivo de mejoramiento.

En este marco, se entenderá la innovación tecnológica como el proceso de rediseño tecnológico y productivo sobre la base de la incorporación y aplicación articulada de invenciones tecnológicas viables, factibles y exitosas, resultado de la experimentación, la investigación, el desarrollo, y la recreación de nuevos conocimientos y saberes, adaptados para una nueva gestión de procesos y productos, bienes y servicios; entre ellos y de modo fundamental, el educativo. De este modo, la innovación se diferencia del descubrimiento y de la invención como propuesta inédita eminentemente procedimental o técnica, aunque pueden relacionarse todas ellas en la práctica pedagógica, son de gran utilidad para iluminar la presente investigación.

\section{El paradigma fenomenológico interpretativo}

Para Kuhn (1962), "Un paradigma es lo que los miembros de una comunidad científica comparten, $\mathrm{y}$, recíprocamente, una comunidad científica consiste en hombres que comparten un paradigma" (p. 33). A partir de sus publicaciones el termino paradigma ha provocado numerosas reflexiones en torno a su significado y alcances, representando una manera de como visualizar el mundo, de explicar y comprender la realidad. Aunque parece irreconocible entre sí, debido a las distintas maneras de concebir el propósito de la investigación, la relación del docente con los estudiantes, los hechos y los valores, la tecnología y la educación.

Sin embargo, la tendencia actual parece orientarse hacia la complementariedad metodológica, restándole importancia a diferencias que se establecen en lo ontológico o en lo epistemológico. En este sentido, Coolican (2005), establece que "El análisis fenomenológico 
interpretativo intenta describir la perspectiva y comprensión que un individuo tiene del mundo y al mismo tiempo reconoce la función constructiva del investigador en la interpretación de la experiencia de ese individuo". (p. 2). El análisis se presenta como una reacción explicativa y detallada del investigador ante la situación problemática de estudio, aunando la definición de Kuhn, se percibe el paradigma fenomenológico interpretativo, donde la experiencia y aplicación de las tecnologías, permiten visualizar e interpretar los desarrollos cognitivos en los estudiantes de educación media general.

\section{Metodología}

La investigación se desarrolló bajo el enfoque cualitativo definido por Bonilla y Rodríguez (1997), como un estudio que "intenta hacer una aproximación global de las situaciones sociales para explorarlas, describirlas y comprenderlas de manera inductiva... a partir de los conocimientos que tienen las diferentes personas involucradas en ella... esto supone que los individuos interactúan”. (p. 70). El artículo se basa en el análisis de las influencias cognitivas que se proporcionan en los estudiantes, como personas involucradas en el objeto de estudio, mediante sus herramientas tecnológicas que definen la estructura ambiental, dejando como segundo plano las interacciones socioeducativas, para analizar los cambios y avances del desarrollo cognitivo. Desde una perspectiva axiológica, el análisis se desarrolló mediante el enfoque introspectivo vivencial, según Padrón (1998):

...se concibe como producto del conocimiento las interpretaciones de los simbolismos socioculturales a través de los cuales los actores de un determinado grupo social abordan la realidad (humana y social, fundamentalmente). Más que interpretación de una realidad externa, el conocimiento es interpretación de una realidad tal como ella aparece en el interior de los espacios de conciencia subjetiva (de ahí el calificativo de Introspectivo). Lejos de ser descubrimiento o invención, en este enfoque el conocimiento es un acto de comprensión. (p. 4)

Desde esta perspectiva de estudio, se desenvolvió una clara concepción y captación de la información, a través de la interpretación, el dialogo, y la observación que los estudiantes expresan con sus palabras, sus silencios, acciones o gestos.

Para la recolección de información se utilizó la observación de tipo moderada, según Spradley (1980), “el investigador mantiene un balance entre estar dentro y fuera”. (p. 74). De esta manera, en el campo investigativo, la observación se entiende como proceso deliberado, sistemático, dirigido a la obtención de información en forma directa del contexto donde se tuvo lugar las acciones, laborando en la parte interna y externa de aula, como también en el ambiente digital con los estudiantes, para la toma de notas eventualmente al compartir y orientar la realización de las actividades.

Según la modalidad de investigación cualitativa, se empleó la entrevista semiestructurada, la cual para Arias (2012), consiste en "una guía de preguntas, el entrevistador puede realizar otras no contempladas inicialmente. Esto se debe a que una respuesta puede dar origen a una pregunta adicional o extraordinaria". (p. 74). La secuencia de las preguntas y la flexibilidad de la 
formulación, facilitaron la obtención de la data de forma adecuada, para los estudios de forma interpretativa.

\section{Resultados y discusión}

La investigación realizada tanto presencial como a distancia, forjo resultados significativos al aplicarse el espacio virtual de aprendizaje, desarrollándose como un escenario para superar dificultades y temores en la práctica de las ciencias matemáticas, al nivel de educación universitaria, la cual brindo un ambiente de seguridad, confianza y de empatía para manifestar libremente sus conocimientos, tales experiencias, asegurándose que los demás participantes no lo censurarán por ello, sino que, por el contrario, lo adolescentes comprenden y pueden apoyar en la búsqueda de soluciones. Los jóvenes demostraron en el estudio gran acuerdo en que su aprendizaje se desarrolló desde un ambiente cálido, ameno y social, que promovió un clima de confianza para participar.

Ahora bien, algunos autores como Salmon (2004), “destacan la necesidad de una fase inicial social para familiarizar y crear puentes entre los entornos culturales, sociales y de aprendizaje de los participantes”. (p. 126). Sin embargo, desde la investigación se consideró que ese puente socio educativo, fue de construcción continua durante todo el proceso de aprendizaje, y no únicamente en el inicio. Si bien se puede entender que al comenzar una experiencia de formación se debe configurar un espacio para la presentación, a lo largo de las discusiones es cuando se consolida el sentido de identidad con las actividades educativas, y por ello debe dedicarse esfuerzos a lo largo del proceso para la manifestación abierta y espontánea de sentimientos, emociones y agrado al mundo de las matemáticas.

En la investigación se apreció en los estudiantes la crisis de identidad, concebida por Carlson (1985), como una "creencia de que el comportamiento aberrante, extravagante y alocado forma parte de la confusión adolescente o que la depresión es reactiva a una de las muchas vicisitudes de la adolescencia" (p. 71). En la etapa del desarrollo del adolescente, el estudiante atraviesa un momento existencial que lo precisa, viéndose en la necesidad de componer varias imágenes de sí mismo en una sola; como amigo, joven, estudiante, líder, hombre o mujer, aparte de tener que elegir una carrera y estilo de vida. Bajo la incursión de las TIC se nivelo el desarrollo personal, de redefinirse y de poner a prueba la confiabilidad y la afirmación de sí mismo, en el encuentro de su propia identidad, piedra angular en la construcción de su yo.

Las TIC como herramientas de desarrollo tecnológico y actual, permitieron a los adolescentes interrelacionarse con el ambiente virtual, debido a que se encuentran en una etapa final de desarrollo corporal, llevándoles a un mismo nivel de adquisición con sentido de confianza básica, independencia, iniciativa, voluntad y logro de actividades numéricas. Con seguridad su desarrollo biológico y físico tiene que ver con las emociones y angustias existenciales. La búsqueda de identidad resulta afectada naturalmente por estos cambios de orden somáticos. Los adolescentes verificaron sus desarrollos cognitivos en la búsqueda de información y manipulación. Recurriendo al espejo, a través de sus producciones o buscando entre sus compañeros, equivalentes de su actividad. Los cambios biológicos suscitan tal desconcierto que el adolescente recurre al espejo frecuentemente, como para reconocerse, comprobar su propia imagen y estabilizar su aprendizaje. 
Como influencia esencial en esta etapa se resolvió exitosamente que los jóvenes comprendieran quiénes son y qué quieren. Además, la identidad representa una condición indispensable para ejercer con propiedad, decisiones pertenecientes al mundo adulto; para encarar tareas que consumen una carga emocional importante que precisa al adolescente. En las actividades a distancia, se mantuvo un foro abierto para mantener el discurso educativo durante todas las actividades, que inicialmente permitió el conocimiento de unos con otros, pero que posteriormente fue escenario para la expresión de pensamientos, sensaciones, emociones, motivaciones. Semana a semana los adolescentes accedían al foro donde se discutía el tema de aprendizaje y, al mismo tiempo, accedían al foro para dejar comentarios a sus compañeros de aula.

Por otra parte, los participantes demostraron una necesidad afectiva de que se satisface en un grupo. La inscripción y aplicación de las actividades virtuales de las matemáticas responde, además de una necesidad cognitiva, a una necesidad personal de estar comunicado con otros para evitar la soledad de entorno educativo, de socializar su vivencia, contar, demostrar sus prácticas y escuchar las de otros. La presencia afectiva se configura desde relaciones informales a formales y espontáneas, del encuentro entre adolescentes, que atenúa la distancia entre los compañeros y genera entusiasmo por participar e interactuar de manera permanente.

La influencia del sentimiento de ser integrante de una comunidad matemática de aprendizaje virtual fue unos de los elementos más destacados en el estudio. La manifestación de agrado y satisfacción de los estudiantes en ese espacio de formación constituye una de las características más relevantes del análisis. Un alto nivel social y emocional hacia la comunidad desde sentimientos expresados en los mensajes del foro, desde las dificultades hasta los avances en las practicas numéricas, destacando la emoción por participar de forma diferente, por pertenecer a la comunidad, la cohesión grupal, la empatía y la necesidad del otro para el aprendizaje.

Este sentido las TIC no resulta necesariamente de manera espontánea, sino que se reconoce que la creación de estrategias y escenarios intencionados favorece la conformación del sentido de comunidad. La participación estudiantil se consideró frecuente, ya que incidió en los niveles de interacción cognitiva y, por tanto, se relaciona con el diseño e intencionalidad, como parte de la investigación para indagar y prever las influencias que proporcionan el uso de las tecnologías, promoviendo lazos emocionales que permitieron la conformación de un ambiente matemático intelectual, abierto y estimulante.

Los problemas matemáticos virtuales, generaron conflictos cognitivos que orientaron al colectivo estudiantil a la búsqueda de informaciones, recuperación de experiencias vividas y suposición de ideas, permitiendo comprender la complicación de las imágenes geométricas, y así buscar soluciones para construir un pensamiento integrado, aplicando soluciones sistemáticas y empíricas. En las experiencias estudiadas se observó que cerca de la mitad del total de los mensajes contenían evidencias de indicadores de interacción cognitiva, que permite valorar que efectivamente se desarrolló un proceso de construcción de conocimientos. No obstante, el tipo de interacciones cognitivas estuvo centrado en los niveles iniciales de pensamiento crítico, destacando declaraciones sobre hechos desencadenantes como punto de partida de las 
discusiones tales como preguntas, dudas, problemas y declaraciones para la exploración de ideas, conceptos y soluciones de los ejercicios planteados.

Las evidencias de aportaciones centradas en elaboraciones conceptuales producto de la integración de las contribuciones presenciales y a distancia, fueron diferentes, así como aportaciones que revelaran conciencia meta cognitiva, demostrando una situación, en la que fuese necesaria la presencia del facilitador que oriente al grupo hacia tendencias como resolver las primeras fases de pensamiento y dejar al margen actividades de razonamiento a nivel cognitivo. Se puede afirmar que, aunque se observó en las actividades estudiadas un interés por el aprendizaje, por la construcción de conocimiento en colaboración (dados el carácter interactivo de los mensajes), se hace necesario dedicar mayores esfuerzos a la promoción de una semántica ontomatemática en los adolescentes que implique el desarrollo de procesos más complejos de pensamiento, y así su aprendizaje virtual le lleve más allá de sus posibilidades actuales a través de actividades retadoras de alto nivel cognitivo.

Se observó el valor formativo que tiene la mediación por pares académicos, es decir entre la tecnología, la psicología y las matemáticas, cada vez se obtenían más indicios de que el aprendizaje es un proceso social y no individualmente solitario; además, la magnitud de los problemas que enfrentan los adolescentes en su cotidianidad reclama el trabajo investigativo en colectivo; por tal razón, es necesario que el aula de clases de matemática sea un escenario para el aprendizaje virtual de ciudadanía; una acción que coadyuva al logro de esta meta es la participación colectiva en procesos de aprendizaje; de manera tal que las actividades deben predominar, en la medida de lo posible, las experiencias de aprendizaje colaborativo.

La mayoría de los problemas complejos demandaron el talento de los participantes, así que los problemas a ser planteados, en el contexto de la matemática, se debía ofrecer a los estudiantes oportunidades para que: diseñaran planes de acción para abordarlos; discusión de la idoneidad y viabilidad de las acciones planeadas; examinación continua de sus posibilidades reales de ponerlas en juego; formulación de preguntas y cuestionamientos; y, organización estratégica de los recursos (cognitivos, materiales, documentales, tecnológicos) de los que se dispuso para llevar a cabo para la tarea resolutoria. Todo esto exigió en privilegiar el trabajo en equipo ya que éste no sólo permite el desarrollo de habilidades cognitivas, sino que, además, es una manera muy efectiva de aprender y así poder visualizar las cualidades que las TIC permiten avanzar de forma significativo en el razonamiento lógico matemático, mediante la comunicación con otros compañeros.

La idea básica de esta investigación fue aportar bases fundamentales para aportes teóricos, y así sostener que la inteligencia se construye y evoluciona debido a la interacción que el individuo une con el medio tecnológico. Cada etapa del desarrollo muestra un tipo de inteligencia que le caracteriza. El adolescente ha llegado a lo que Piaget llama etapa de las operaciones formales. Ha este nivel del desarrollo cognoscitivo el adolescente utiliza métodos o estrategias de pensamiento diferentes a las empleadas por los niños en etapas anteriores. El pensamiento adolescente se caracteriza fundamentalmente porque está en capacidad de trabajar en forma abstracta y de aventurarse en las formulaciones hipotéticas. 
Para el desarrollo y análisis introspectivo vivencial, el tiempo influye en los niveles de construcción de conocimiento. Para la promover del desarrollo cognitivo en los adolescentes se requiere espacios de tiempo entre 6 y 7 semanas, o, bien, un acuerdo de aportación de mayor frecuencia semanal (de al menos tres o cuatro aportes), de manera de garantizar que las primeras actuaciones destinadas a razonamiento lógico, al reconocimiento y exploración de los problemas, no limiten el paso a las siguientes e importantes fases de integración de conceptos orientados a la resolución del problema y a reflexiones meta cognitivas de mayor nivel de abstracción.

\section{Conclusiones y recomendaciones}

A manera de conclusión, se pueden presagiar de manera futurista, que los estudiantes egresados del bachillerato, como los porvenires dirigentes de la sociedad; deben tomar en cuenta el carácter dinámico del conocimiento, es entendible por qué es necesario interpretar y comprender las diferentes influencias que perciben los estudiantes al enseñarles matemática hoy en función de las exigencias que les hará el entorno científico y tecnológico a sus inicios del mañana a nivel universitario. La efectividad de las TIC no es sólo concebida por su utilidad para la capacitación acreditada y técnica, existen razones intrínsecas a esta disciplina que justifican su enseñanza para el desarrollo cognitivo en los futuros profesionales. En efecto, se ha podido comprobar que entre los motivos que tienen quienes tornan a la matemática como profesión, está la manera como la han aprendido y esto, a su vez, tiene mucho que ver con la forma como le ha sido enseñada; de aquí que el modo como se conduzca el proceso de enseñanza y aprendizaje de la matemática en los diferentes niveles es un factor de gran impacto sobre la vinculación con esta ciencia que, en un futuro, tendrán los estudiantes.

Por otro lado, se puede plantear que, en comparación con el total de la población docente, los especialistas en las diferentes disciplinas siempre han sido en acatamiento; por ello no hay que orientar la enseñanza de la matemática como si todos los estudiantes, en el futuro, la tornarán como la especialidad de su carrera; así que es imprescindible determinar cómo enseñarla de modo que contribuya a la formación integral del ciudadano del futuro; de este modo hay que concebir la aplicabilidad de las TIC para fortificar la capacidad intelectual del adolescente en la matemática, no como un fin en sí mismo, sino como un medio para contribuir al desarrollo cognitivo e integral de la personalidad del joven. Por tanto, en la enseñanza de las ciencias numéricas deben tenerse presentes aspectos motivacionales, tomando en cuenta que la forma como se enseña la disciplina, y no sólo los contenidos en serie tienen una gran incidencia sobre las elecciones vocacionales.

Así que quien enseña matemática, al nivel de educación universitaria, tiene una gran incidencia sobre las vinculaciones que, en el futuro, sus estudiantes querrán tener con la matemática; ante ésta, se afina que los docentes tienen dos opciones, una es considerarla como una ciencia hecha, en la que nada es modificable, o como una ciencia por construir, en la que es posible reinventar aquello que es conocido por quienes la dominan, pero que aún no es dominada por quienes están intentando aprenderla.

Cabe indagar que los adolescentes, al aplicar las TIC pueden disponer de un tiempo suficiente para activar todos sus mecanismos de funcionamiento intelectual cuando les corresponde enfrentarse al proceso de búsqueda de solución a un problema de Matemática. Al 
contrario, en cuanto la actitud del docente ante el trabajo de los estudiantes. Uno de los aspectos centrales de la matemática actual es la consideración del estudiante como un constructor y desarrollador inerte de su nivel cognitivo ajustado a su propio aprendizaje de la matemática ello implica que ha de brindársele espacios de libertad en su relacionamiento y conectivismo digital con los objetos y proceso matemáticos de los que se ha de apropiar con miras a desarrollar su formación matemática propia.

Por ello, desde el punto de vista introspectivo vivencial el docente de matemática debe brindar al adolescente situaciones de aprendizaje basadas en el planteamiento de problemas de su experiencia que le resulten atractivos, y respetar las reacciones que los estudiantes tengan ante ellos; apreciar, valorar y respetar las vías que el joven seleccione para abordar los problemas planteados; además, el orientador debe resistirse a la tentación de sustituir los esfuerzos del alumnado por los suyos propios; y, por el contrario, estimularle para que persevere en las buenas ideas que se le ocurran para solucionar un problema; una vez que el alumno ha arribado a alguna solución, el docente debe intervenir con miras a orientar el proceso de formalización y de reflexión sobre la actividad realizada con el fin de mediar el proceso de transferencia, generalización y desarrollo cognitivo.

Para todo docente universitario aducir o atreverse a inventar, a innovar, a ensayar nuevos enfoques para la enseñanza de la matemática, sobre todo si toma en cuenta los negativos resultados a los que les ha conducido la enseñanza tradicional, le ha de considerar muy dificultoso. Como se ha expuesto, las influencias cognoscitivas rompen la enseñanza tradicional de matemática, que siguen el esquema de exposición de la teoría que traen consigo los estudiantes desde el bachillerato, como también la muestra de ejemplos; realización de ejercicios en la clase; y asignación de deberes para el hogar; todas estas acciones siempre han estado a cargo del docente; al alumno le corresponde hacer los ejercicios en clase y efectuarlos en su residencia.

Las TIC presentan cambios profundos tanto en el contenido a ser enseñado como en las prácticas que los docentes de matemáticas, que ponen en juego las aulas virtuales de esta asignatura. De modo que estas atribuciones que admite el estudiante le lleva a romper bajo nivel de desempeño matemático, aburrimiento de éstos y, en los casos más graves, la matematefobia o aversión hacia esta ciencia. Estas anomalías fueron superadas al introducirse nuevos modos de aprendizaje basado en la investigación, dando responsabilizad a los estudiantes por su propio aprendizaje, bajo un estudio educativo, especialmente desde las ciencias cognoscitivas.

\section{Bibliografía}

Arias, F. (2012). El proyecto de investigación. Editorial Episteme. Caracas.

Ausubel, D.P. (1976). Psicología educativa. Editorial. Trillas, México.

Ausubel, D.P., Novak, J.D., y Hanesian, H. (1983). Psicología Educativa: Un punto de vista cognoscitivo. 2da edición. Editorial Trillas. México.

Bonilla, Elssy. y Rodríguez, Penelope. (1997). La Investigación en Ciencias Sociales. Más allá del dilema de los métodos. Segunda edición. Bogotá, Colombia. Grupo Editorial Norma. 
Cabada Arenal, M. T. (2001). Estudio del paradigma tecnológico y su repercusión en la formación de los profesionales de la información. Vol 9. ACIMED. Disponible: http://bvs.sld.cu/revistas/aci/vol9_3_02/aci08301.pdf

Cabero Almerana, J. (2005). Las TICs y las Universidades: retos, posibilidades y preocupaciones. Revista de la Educación Superior, 34(3):77-100, (ISSN 0185-2760). Disponible: http://tecnologiaedu.us.es/bibliovir/pdf/Las\%20TICs\%20y\%20las \%20 Universid ades.pdf (Consultada 24/03/07).

Carlson G.A. (1985). Visión global de las formas enmascaradas alternativas a la depresión. En DP Cantwell y GA Carlson. Trastornos afectivos en la infancia y la adolescencia. Martínez-Roca, Barcelona.

Padron, J. (1998). La estructura de los procesos de investigación. Universidad Nacional Abierta. Disponible: $\quad$ http://dip.una.edu.ve/mae/978investigacioneducativa/paginas/L ecturas/UNIDAD\%204/Padron-LaEstructuradelosProcesosdeInvestigacion.pdf

Coolican, H. (2005). Métodos de investigación y estadística en psicología. Editorial manual moderno. México. Disponible: http://www.franciscohuertas.com.ar/wpcontent/uploads/2011/04/IT_Coolican.pdf

Fainholc, B. (2012). Una tecnología educativa apropiada y crítica. Editorial Lumen. Buenos aires, Argentina.

Calle, L. (2011). Estilos de Aprendizaje e identificación de actitudes y variables vinculadas al uso de las TICs en los alumnos de Enfermería de la Universidad de Salamanca. Tesis doctoral. Universidad de Salamanca. España

Gardner, H. (2011), Cultura de Pensamiento. Proyecto Zero, Harvard University, USA.

García, M. A. (2010). Conformación de comunidades de aprendizaje en red. Universidad Central de Venezuela. Venezuela.

Kuhn, T. (1962). La estructura de las revoluciones científicas. Fondo de Cultura Económica. México.

Landeau, R. (2012). Metodología y nuevas tecnologías. Editorial Alfa. Caracas, Venezuela.

Novak, J.D. (1998). Conocimiento y aprendizaje. Los Mapas conceptuales como herramientas facilitadoras para escuelas y empresas. Alianza. Madrid.

Pírela, I., Gómez, R. y González, P. (2002). La psicología educativa. Universidad nacional abierta. Caracas, Venezuela.

Salmon, G. (2004). E-actividades: el factor clave para una formación en línea activa. Barcelona: UOC.

Spradley, J. P. (1980). Participant observation. New York: Holt Rinehart and Winston. 